\title{
LOS MENORES Y LAS SERIES DE FICCIÓN: INVESTIGANDO CON LOS SERRANO
}

\section{CHILDREN AND TV SERIES: RESEARCHING WITH LOS SERRANO}

\section{AUTORES}

\section{Miriam Alonso Bayón}

Cadena COPE Valladolid

nairim69@hotmail.com

Joaquín Sotelo González

Universidad Complutense de Madrid

joaquin.sotelo@gmail.com

\section{RESUMEN}

A pesar de la aparición de los nuevos fenómenos de comunicación venidos de la mano de las nuevas tecnologías, le televisión continúa siendo el rey de los medios de comunicación de masas. Las series de ficción representan hoy uno de los contenidos preferentes de este medio y la audiencia infantil y juvenil, uno de sus principales targets. El presente artículo muestra los resultados de una investigación académica realizada con menores y una de las series de ficción españolas de mayor éxito: Los Serrano.

\section{PALABRAS CLAVE}

Menores - Televisión - Series de ficción - Los Serrano - Grupo de discusión. 


\section{ABSTRACT}

In spite of new communicative phenomena arrived with new technologies television is still the King of the mass media. Moreover TV series are today one of the most popular audiovisual products and young people represent a priority target for them. This paper shows the results obtained by researching with children and one successful Spanish TV serie: Los Serrano.

\section{KEYWORDS}

Children - Television - TV series, Los Serrano, discussion group.

\section{ÍNDICE}

1 Introducción

2. La serie y el capítulo

3. Investigando con Los Serrano

4. Bibliografía

\section{Introducción}

Vivimos inmersos en una sociedad impregnada de medios de comunicación de alcance masivo en la que la televisión sigue ocupando el puesto más privilegiado en las preferencias -y también en las posibilidades- de la inmensa mayor parte de los ciudadanos. La llegada de nuevos fenómenos comunicativos apenas ha mermado la fuerza y el poder de un medio con el que "interactuamos" cada día y cuyas propiedades casi hipnóticas le siguen confiriendo una enorme e indiscutible capacidad de influencia sobre las personas y sobre los grupos que éstas componen. 
Sin duda, el grupo o sector social más "permeable" a los mensajes emitidos por televisión es el compuesto por los niños y las niñas. Los menores están permanentemente expuestos al fenómeno de "transmisión de conciencia" que ejercen los medios y, en especial, la televisión, que cuela en sus mentes estilos, modas, ídolos, actitudes y creencias.

Los niños ven la televisión incluso antes de saber leer y escribir. A los dieciocho años, como apunta Anthony Giddens, "habrán pasado más tiempo viendo la televisión que haciendo cualquier otra actividad, a excepción de dormir" (Giddens, 2001, 575).

A pesar de las crecientes voces críticas, cada vez son menos los programas dirigidos al público infantil y juvenil, por lo que los menores se ven de alguna forma obligados a ver una programación no expresamente dirigida a ellos y, en muchas ocasiones, potencialmente inapropiada.

Alan M. Rubin (Rubin, 1984) identificó en su día dos tipos de uso de la televisión: el instrumental, guiado por una meta o propósito distintos del mero entretenimiento, y el ritual, asociado al puro entretenimiento. Rubin concluyó que el uso predominante es el instrumental, conclusión que, años más tarde, recogería Yolanda Montero Rivero para apuntar la idea de que "los adolescentes buscan en la pantalla las respuestas a las situaciones reales que les preocupan" (Montero Rivero, 2006, 6667).

Presentamos a continuación los resultados de parte de una investigación en la que hemos estudiado las posibles influencias de un contenido televisual concreto, una serie de ficción española de éxito, Los Serrano, sobre los miembros de un grupo de 10 menores, niños y niñas, de edades comprendidas entre los cuatro y los diecisiete años. La técnica utilizada ha consistido en someter al grupo al visionado de un capítulo de esta serie. Posteriormente, hemos recabado, mediante método test, algunas opiniones de los menores. El capítulo elegido fue el segundo de la primera 
temporada, titulado "Un padre perfecto". A pesar de que en breve comenzará la sexta temporada y de las distintas posibilidades que se nos ofrecían, se eligió el segundo capítulo porque es el primero de la serie en el que los personajes infantiles cobran protagonismo y, por tanto, están muy marcados los rasgos y características que cada uno de ellos desarrollará durante la serie, facilitando así la posible identificación entre los actores y los espectadores.

\section{La serie y el capítulo}

El primer capítulo de la serie Los Serrano, emitido por Telecinco en 2003, nos sirve como introducción para situarnos en el entorno en el que evolucionarán las tramas: Los Serrano son una familia formada a partir del matrimonio de Diego, viudo y padre de tres hijos varones (Marcos, Guille y Currito), y Lucía, divorciada y madre de dos hijas (Eva y Teté). Tras la boda de Diego y Lucía, comienza la convivencia. La nueva familia tendrá que afrontar una serie de conflictos. La serie evoluciona sumando tramas en las que se van integrando el resto de personajes: la abuela Carmen (madre de Lucía), el tío Santiago (hermano de Diego, con el que regenta una taberna) y la familia Martínez (amigos íntimos de los Serrano).

En el segundo capítulo, titulado "Un padre perfecto", ya conocemos a los principales personajes y empiezan las desavenencias. Los chicos y las chicas no se llevan del todo bien y surgen las primeras tensiones de convivencia. Diego, preocupado porque todo vaya bien en casa, intenta cambiar la actitud de sus hijos e incluso va al psicólogo con Lucía para saber cómo gestionar la nueva situación familiar.

Por su parte, Lucía se incorpora a su nuevo trabajo como profesora en el colegio del barrio donde vive la pareja, en el que también trabaja Candela (la mujer de la familia Martínez y la que, a la postre, será su mejor amiga). Lucía será la tutora de la clase en la que están juntos Guille y Teté. 
Guille no quiere que sus amigos sepan que Teté es su hermanastra y que, por lo tanto, Lucía, la profesora, es su madre. Pero todos terminan enterándose cuando Teté lee públicamente una redacción sobre su familia.

Los chicos mayores, Marcos y Eva, son los que mejor relación tienen. Durante el capítulo elegido, Eva solicita permiso a su madre para que su novio, que viene a visitarla desde Barcelona, pueda dormir en casa, pero cuando Lucía y Diego deciden consentirlo, Eva cambia de opinión y dice que prefiere irse con él a una pensión, algo que mantiene muy preocupados a sus padres. Finalmente, Eva recibe una llamada de su novio con la que éste da por concluida la relación. Eva decide salir a despejarse y en la discoteca se encuentra con Marcos (su hermanastro) y con Raúl (el hijo de Candela y amigo de Marcos) que la ayudan a desahogarse.

Esta es la sinopsis del capítulo elegido para el visionado y del que más adelante comentaremos algunas secuencias relevantes, cuando analicemos las respuestas dadas por los menores con los que hemos investigado.

\section{Investigando con Los Serrano}

Tras la proyección del capítulo "Un padre perfecto" en presencia de los diez niños y niñas participantes en el estudio, procedimos al planteamiento público de una batería de preguntas preconcebidas para que todos y cada uno de los menores del grupo investigado respondieran. Estos fueron los resultados y algunas de las reflexiones suscitadas a la vista de los mismos:

1. ¿Ves normalmente la serie Los Serrano?

Siete de los diez menores que participaron en la sesión respondieron que sí ven normalmente esta serie, clasificada como no recomendada para menores de trece 
años. Sólo dos -de los siete menores que respondieron afirmativamente- cumplían el requisito de edad superior a los trece años; el resto de los que afirmaron ver normalmente la serie, no alcanzaban esta edad. Encontramos aquí la primera apelación a la responsabilidad de los padres que permiten que sus hijos vean una serie que no está dirigida específicamente a ellos.

Según el estudio Programación Infantil de Televisión: Orientaciones y Contenidos Prioritarios $(2005,20)$, realizado por el Instituto Oficial de Radio y Televisión, las 22.30 es la hora más vista por la audiencia infantil, a pesar de que no está específicamente protegida para este público.

Actualmente, las series de televisión utilizan una estrategia, que además está funcionando a la perfección, para, entre otras razones, atraer al público infantil y juvenil: incorporar como protagonistas a menores que llamen la atención de esta audiencia. El caso de Los Serrano es el más extremo, ya que, a través de sus personajes, vemos representado a un muy amplio espectro sociológico, que va desde la niñez, hasta la juventud. Además, desde el año 2003 hasta hoy, venimos asistiendo a diversos procesos evolutivos de los personajes infantiles, por lo que sus conductas podrían convertirse más fácilmente en referentes para los menores que siguen la serie y que, de alguna manera, pudieran experimentar algún grado de identificación con esos personajes.

2. ¿Quién es tu personaje preferido? ¿Por qué?

En cuanto a los personajes preferidos por los niños del grupo, los más votados fueron Guille y Teté. La justificación más aludida para elegir al primero fue que "es el más gracioso" y "el más travieso" y quienes eligieron a Teté, que fueron todas chicas, alegaron su belleza para justificar su elección. 
Encontramos el primer ejemplo de que los estereotipos y modelos que la serie transmite funcionan. De las respuestas de los menores, parece deducirse que no guía tanto la elección de su personaje preferido el personaje en sí como la razón por la que lo han elegido como su preferido. Guille es un chico travieso, siempre metido en líos y que, en ocasiones, manifiesta actitudes machistas y, sin embargo, los niños no sólo no han sido críticos con él, sino que aplauden su conducta. Algo similar ocurre con Teté, presentada como una fanática de las compras, de la moda y, por tanto, de la imagen y la estética. Las niñas se sienten atraídas por este personaje, hasta el punto de que algunas piensan y expresan que "ser así es lo más apropiado", y toman a Teté como referencia para su día a día.

\section{3. ¿Te identificas con algún personaje? ¿Con cuál?}

Sólo una chica de los diez menores del grupo afirma sentirse identificada con una de las protagonistas de la serie. Se identifica con Eva, por su edad y por su forma de ser. Observamos que los rangos de edad representados son eficientes, dado que esta chica, de dieciséis años, se identifica con el personaje que más se asemeja a su situación.

4. ¿Te gustaría parecerte a algún personaje?

A esta pregunta, dos chicas respondieron que sí. La primera, de seis años, reconoce que le gustaría parecerse a Teté, que es su personaje preferido. $Y$ a la segunda, de once años, le gustaría parecerse a Eva, que también es su personaje favorito "por ser la más normal".

Cuando un espectador se siente identificado con un personaje es muy común que tienda a imitar sus actuaciones y, sobre todo, su aspecto: "Los espectadores tienden a adoptar sin apenas darse cuenta, los gestos, los peinados, el tipo de ropa y demás signos externos de los actores o presentadores, sobre todo de los favoritos" (Padilla 
Novoa, 2002, 122). Es probable que estas niñas hagan de las protagonistas cierto modelo de conducta.

5. ¿Se parecen tus padres a Lucía y a Diego?

La quinta pregunta, sobre la semejanza entre Lucía y Diego y los padres de los participantes en el visionado, obtuvo nueve respuestas negativas y sólo una afirmativa. Tal vez en este caso, por ser el segundo capítulo de la serie, se dificulta la identificación directa, ya que los estereotipos que los personajes representan están muy marcados $y$, al exagerarse, distan un tanto de la realidad.

Los estereotipos mostrados en la serie son los que describe Ana Guil Bozal: "Las mujeres muestran un comportamiento emocional, representan la intuición, el sentimiento, la pasión, la suavidad, proveen de bienestar a su familia, mientras que los hombres representan el impulso, la frialdad, agresividad y violencia, el dominio" (Guil, 1998, 95-100). Joan Ferrés también señala estas mismas características estereotípicas que se atribuyen a hombres y a mujeres. Las mujeres tienden a aparecer como dependientes, sensibles y pacíficas, mientras que los hombres se muestran como activos, fuertes y violentos (Ferrés, 1997, 161-162). Los personajes de Diego y Lucía cumplen estos estereotipos: los hombres de la serie no son cariñosos entre sí: Diego utiliza las "collejas" para aleccionar a sus hijos, mientras que Lucía opta por el diálogo y el cariño. Ambos trabajan, sin embargo, la taberna familiar que Diego regenta, representa para él mucho más que un simple trabajo, algo que no sucede con tanta intensidad en el caso de Lucía y su trabajo como profesora en un colegio. Y mientras Lucía se preocupa por su aspecto físico, va al gimnasio y cuida su dieta, Diego no lo hace.

Son algunos de los ejemplos de estereotipos más claros que la serie muestra y que pueden influir en el proceso de socialización: "Tanto en series y filmes como en los 
informativos, el proceso de socialización inconsciente puede provenir de una estereotipada selección de personajes y de contenidos, [...]"(Ferrés, 1997, 167).

6. ¿Te fijas en las series para saber lo que tienes que hacer en determinadas situaciones?

Tres de las chicas del grupo reconocieron fijarse en las series para resolver determinadas situaciones de su vida cotidiana.

En series de este tipo, que representan la vida diaria de una familia, se muestran numerosos problemas y conflictos reconocibles y habituales en la vida ordinaria de las personas y los grupos. Joan Ferrés explica cómo se produce el aprendizaje por modelización y el papel que hoy juega la televisión en este ámbito: "Antes, el niño sólo contaba con los modelos que su familia y el entorno más inmediato le concedían, sin embargo, hoy, los medios de comunicación ofrecen una gran cantidad de modelos a seguir" (Ferrés, 1997, 62). Según Ferrés, las conductas que observamos en los medios se convierten en un ejemplo para los espectadores, en general, y mucho más para los menores, que están modelando su manera de ser: "Los modelos observados crearían expectativas de beneficios para determinados tipos de comportamiento y anticiparían consecuencias negativas para otros"(Ferrés, 1997, 63). En cierto sentido, las series podrían establecer indirectamente las futuras conductas de sus espectadores.

7. ¿Tus aficiones se parecen a las de los protagonistas?

Sobre la semejanza entre las aficiones de los protagonistas de la serie y las de los niños y niñas del grupo investigado, siete respondieron que sí existe similitud. Las aficiones representadas por los chicos y las chicas de la serie están muy bien diferenciadas. A los protagonistas les gusta el fútbol, los videojuegos y hacer travesuras; mientras que las actividades preferidas de las protagonistas son ir de 
compras, estar con sus amigas y la lectura. Éstas actividades preferentes parecen atribuidas siguiendo un patrón muy estereotípico por el que los deportes y videojuegos son propiamente varoniles y las aficiones relacionadas con el cuidado personal y aquellas para las que no se necesita demasiada energía ni fuerza física parecen más propias de mujeres. Más adelante, veremos cómo asumen estos estereotipos los menores.

\section{8. ¿Crees que el fútbol es cosa de chicos?}

Cinco de los diez menores investigados respondieron con un rotundo sí a esta pregunta. $Y$ tres fueron mujeres. Una de las razones del elevado número de respuestas afirmativas a esta pregunta podría deberse a la propia influencia del capítulo proyectado, en el que se repiten con insistencia ideas como que, en la casa, lo más importante es que los hombres vean el fútbol juntos y las repeticiones de las mejores jugadas, pero que desde que hay mujeres eso está cambiando. También se habla de que el fútbol, los tacos y orinar la tapa del retrete son cosas propias de hombres, por lo que creemos que las ideas transmitidas al respecto han podido guiar las respuestas afirmativas a la pregunta de si el fútbol es cosa de chicos, especialmente en el caso de las chicas más pequeñas del grupo, de 6 y 8 años, que respondieron sin dudar que el fútbol es cosa de chicos. Por otra parte, los dos chicos que respondieron que el fútbol es para chicos, reafirmaron también ciertos prejuicios que se muestran reforzados en el capítulo.

9. ¿Y las gamberradas... son propias de chicos o de chicas?

Las preguntas nueve y diez siguen la misma temática que la anterior, la "guerra de sexos". A propósito de las gamberradas, hemos obtenido los siguientes resultados: cinco personas, chicos y chicas, creen que las gamberradas son más propias de chicos; sólo una dice que son cosa de chicas y tres afirman que tanto chicos como chicas "pueden" hacer gamberradas. Una persona se abstuvo de responder. Las 
respuestas están algo más equilibradas que en la anterior pregunta, pero la que predomina, la que dice que las gamberradas son más propias de chicos, es armoniosa con la representación que ofrece la serie en este aspecto, en la que los "expertos en gamberradas" son los chicos y, por encima de todos, el personaje de Guille.

10. ¿Crees que las chicas limpian más que los chicos?

En esta pregunta hay una mayoría aplastante que responde que las chicas limpian más que los chicos. Sólo una persona opinó que esto no es así. En la serie, la idea mostrada es precisamente ésta, ya que, como se escucha en una secuencia del capítulo, "desde que en la casa Serrano hay mujeres, todo está más ordenado y limpio" y los propios protagonistas masculinos afirman que "las chicas limpian más".

\section{1. ¿Es malo estar gordo?}

En el capítulo utilizado para llevar a cabo nuestra investigación se habla en varias ocasiones, de forma directa e indirecta, acerca del peso de varias personas. Lucía y Candela reconocen acudir regularmente al gimnasio para mantener su forma y su peso. Diego no conoce el nombre de los amigos de su hijo y a uno de ellos lo distingue del resto refiriéndose a él como "el amigo gordo". Además, en una escena en la que aparecen los chicos sentados en el sofá viendo las jugadas de un partido de fútbol, Santiago critica a uno de los jugadores llamándole "gordo". Pero, la secuencia en que más hincapié se hace sobre este asunto es en la que Yoli, la hermana de José María ("el amigo gordo de Guille"), lee una redacción sobre su familia en la que argumenta que en su casa todos son gordos, menos su padre, del que dice que les esconde la comida y que, cuando cree que nadie le escucha, exclama "ipor qué no serán como yo!". 
Tras ver el capítulo, cinco de los diez menores del grupo contestaron que "no es malo estar gordo"; tres dijeron que sí y dos creen que "es malo, pero no demasiado". Entre los participantes no había ningún miembro con problemas aparentes de peso, pero nos preguntamos qué habría pasado si un niño con problemas de este tipo hubiera visto y escuchado las opiniones que se estaban manifestando sobre este tema. Es muy probable que se hubiera sentido aludido y dañado.

\section{2. ¿Tienes música de Fran Perea? ¿Y de Santa Justa Klan (SJK)?}

Hablamos ahora de los negocios surgidos a partir de la serie, la denominada "producción cruzada". Cuando la serie comenzó, Fran Perea, Marcos en la ficción, sacó un disco a la venta en el que incluía las canciones que cantaba en la propia serie. Fue un éxito, aunque desde entonces no ha vuelto a editar ningún disco más.

En el año 2006, y bajo la denominación grupal de $S / K$, Guille, Teté, Boliche y Dvd grabaron un disco, y recientemente han sacado a la venta su segundo $C D$. Además, hay toda una gama de productos corporativos de $S J K$ a la venta: camisetas, bolsos, chapas,fotos...

Observamos que alrededor de Los Serrano se ha creado una gran red de exitoso marketing entre los muchos fieles seguidores de la serie. Cinco de los menores preguntados tienen música de Fran Perea y/o de $S / K$, es decir, la mitad de los participantes "consumen serie" más allá de la simple emisión. Cabe destacar el caso curioso de una niña de once años que no sigue de manera habitual la serie, pero sí tiene música de sus protagonistas, lo que evidencia el éxito de la misma y la eficacia mercadotécnica de sus productores.

13. ¿Puedes decirme de qué marca es la leche que toman Los Serrano? ¿Y la de la gomina que utilizan? ¿Sabes qué bebía Marcos en la escena del salón? 
Esta serie de preguntas guarda relación con el fenómeno conocido como product placement o "emplazamiento de productos", algo muy común en las series de éxito de la televisión y que consiste en hacer publicidad "indirecta" de marcas y/o productos.

En este capítulo de Los Serrano, encontramos marcas como Adidas (la ropa que viste Lucía en el gimnasio) o Nike (marca de la que Raúl viste una camiseta en el entrenamiento), Coca-Cola (podemos ver un primer plano de Marcos bebiendo este refresco), Giorgi (bote de gomina en el baño) y Puleva (leche que toma la familia Serrano para desayunar). Debemos tener en cuenta que el capítulo del que hablamos fue el segundo de la serie y ya contaba con todo este abanico de marcas para publicitar. Actualmente, que la serie ya disfruta de un notable éxito consolidado, el número y la presencia en pantalla de marcas y productos han aumentado considerablemente. Por ejemplo, la marca de jamones Navidul aparece siempre que la taberna es el escenario.

De toda la batería de cuestiones planteadas al grupo de menores con el que hemos trabajado, las preguntas sobre product placement son las que menos se han respondido. Ningún niño acertó la marca de leche que se había visto y sólo uno adivinó el nombre de la gomina que utilizaban los personajes; sin embargo, la mitad, cinco, identificaron la marca del refresco que Marcos bebía en una de las escenas del capítulo. 


\section{Bibliografía}

DEL Río ÁlVAREZ, Miguel y ROMÁN BLAS, Mariano (eds.) (2005): Programación Infantil de Televisión: Orientaciones y Contenidos Prioritarios. IORTV. Madrid.

FERRÉS, J oan (1997): Televisión subliminal. Socialización mediante comunicaciones inadvertidas. Paidós. Barcelona.

GIDDENS, Anthony (2001): Sociología. 4ạ Edición. Alianza Editorial. Madrid.

GUIL BOZAL, Ana (1999): "El papel de los arquetipos en los actuales estereotipos sobre la mujer", en Comunicar, 11: 95-100.

MONTERO RIVERO, Yolanda (2002): Televisión, valores y adolescencia. Gedisa. Barcelona.

PADILLA NOVOA, Manuel (2002): Técnicas de persuasión en la televisión. Ed. Del Laberinto S.L. Madrid.

RUBIN, Alan M. (1984): "Ritualized and Instrumental Television Viewing", en Journal of Communication, 34 (3): 67-77. 